\title{
Design of field limiting ring employing trench structure for high power devices
}

\author{
Jong-Seok Lee and Man Young Sung a) \\ Dept. of Electrical Engineering, Korea University, \\ 5-ga Anam-Dong, Seongbuk-Gu, Seoul 136-701, Korea \\ a)semicad@korea.ac.kr
}

\begin{abstract}
Excellent voltage blocking capability and reliability can be obtained by trenching the field-limiting ring site which would be implanted. The trench etch step makes the junction depth deeper so that junction curvature effect and surface breakdown are less happened. The numerical analyses reveal two facts that the trenched field limiting ring has smaller maximum electric field and the electric field peak is deeper from the substrate surface, hence silicon dioxide layer can be protected. Therefore the voltage blocking capability and reliability of the new structure can be improved. The simulated results show that the trenched field limiting ring can have smaller critical electric field and accomplish near 30\% increase of breakdown voltage in comparison with the conventional structure.
\end{abstract}

Keywords: edge termination, trench, field limiting ring

Classification: Electron devices, circuits, and systems

\section{References}

[1] B. J. Baliga, Power Semiconductor Devices, first ed., PWS, 1996.

[2] S. M. Sze and G. Gibbons, "Effect of junction curvature on breakdown voltage in semiconductors," Solid-State Electronics, vol. 9, no. 9, pp. 831845, Sept. 1966.

[3] M. S. Adler, V. A. K. Temple, A. P. Ferro, and R. C. Rustay, "Theory and breakdown voltage for planar devices with a single field limiting ring," IEEE Trans. Electron Devices, vol. 24, no. 2, pp. 107-113, Feb. 1977.

[4] J. S. Lee, E. G. Kang, and M. Y. Sung, "Shielding region effects on a trench gate IGBT," Microelectronics Journal, vol. 39, no. 1, pp. 57-62, Jan. 2008.

[5] E. G. Kang, S. H. Moon, and M. Y. Sung, "A new trench electrode IGBT having superior electrical characteristics for power IC systems," Microelectronics Journal, vol. 32, no. 8, pp. 641-647, Aug. 2001.

[6] D. C. Sheridan, G. Niu, J. N. Merrett, J. D. Cressler, J. B. Dufrene, J. B. Casady, and I. Sankin, "Comparison and optimization of edge termination techniques for $\mathrm{SiC}$ power devices," Proc. 13th International Symposium on Power Semiconductor Devices and ICs, 2001, ISPSD '01, pp. 191-194, 2001. 


\section{Introduction}

Power semiconductors are widely used as Inverters, high voltage switches and Motor Drivers, etc [1]. The blocking voltage is one of the most important parameters for these power semiconductors [2]. In actual power semiconductors utilizing planar diffusion technology, however, the high-voltage junction must intersect the surface at some finite position [3]. The resulting junction curvature compress the equi-potential lines where the junction bends to the surface and increase the maximum electric field. In order to improve the breakdown characteristic and the reliability, it is useful to shift the point of maximum electric field from the surface to the substrate interior and to reduce the peak value $[4,5]$.

The field limiting ring, Fig. 1 (a), has been widely used in Si technology as an effective means of reducing the amount of field crowding at the main junction by spreading the depletion layer past consecutively lower potential floating junction rings [6]. However, the conventional field limiting ring suffers from an oxide reliability problem due to the high electric field peak near the silicon-oxide interface.

In this paper, the trenched field limiting ring is proposed to increase breakdown voltage and improve reliability problems simultaneously. The newly proposed structure has deep ring junctions and they can be obtained by trenching the field limiting ring site which would be ion-implanted as can be seen from the Fig. 1(b). As the junction depth is deeper, the junction curvature is larger and the electric field peak is smaller. By adding a etch step, the edge effects caused by junction curvature can be deceased and the

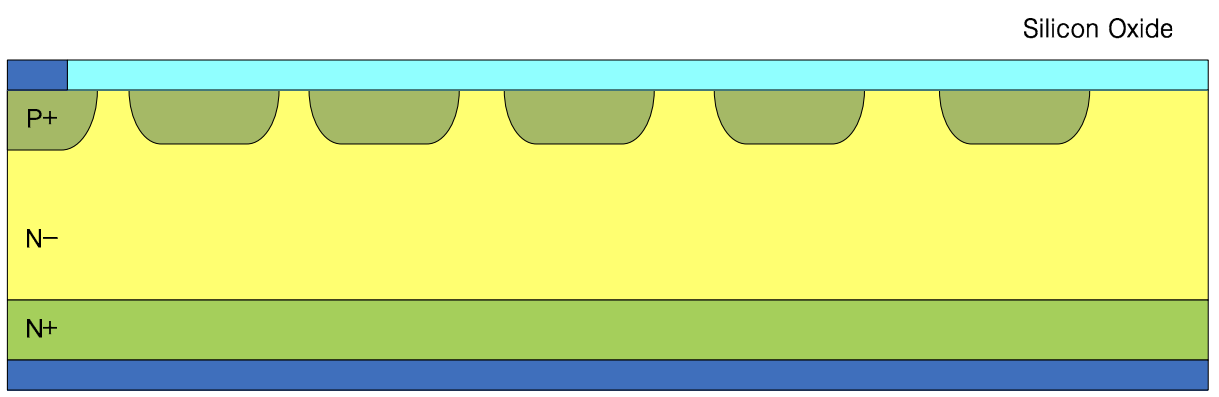

(a)

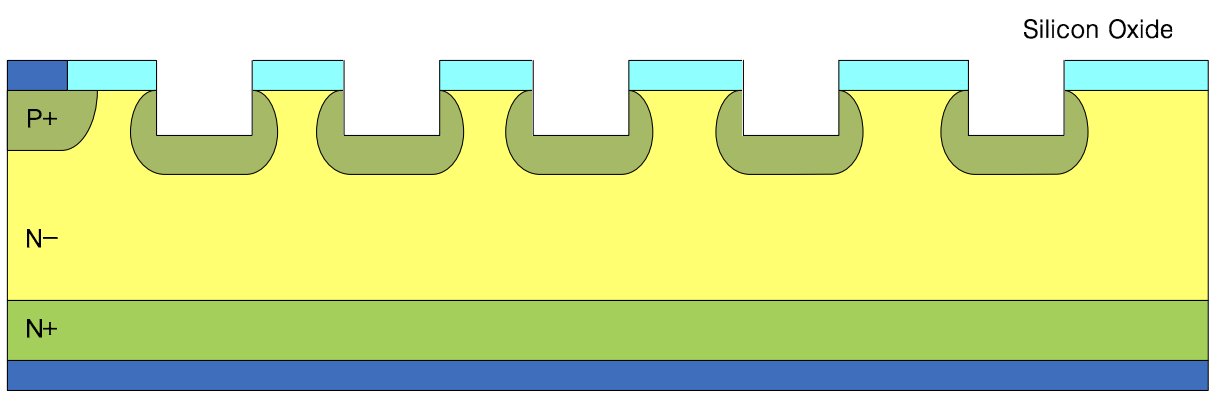

(b)

Fig. 1. (a) Conventional field limiting ring, (b) Trenched field limiting ring. 
breakdown voltage can be increased.

\section{Trench field ring simulation results}

In this section, the trenched field limiting ring for $1,700 \mathrm{~V}$ power devices is designed and measured by TMA MEDICI simulator to confirm the expectations.

A conventional 5-ring structure and trenched 5-ring structure are designed. The potential distributions of these two structures are showed in Fig. 2 (a), (b).

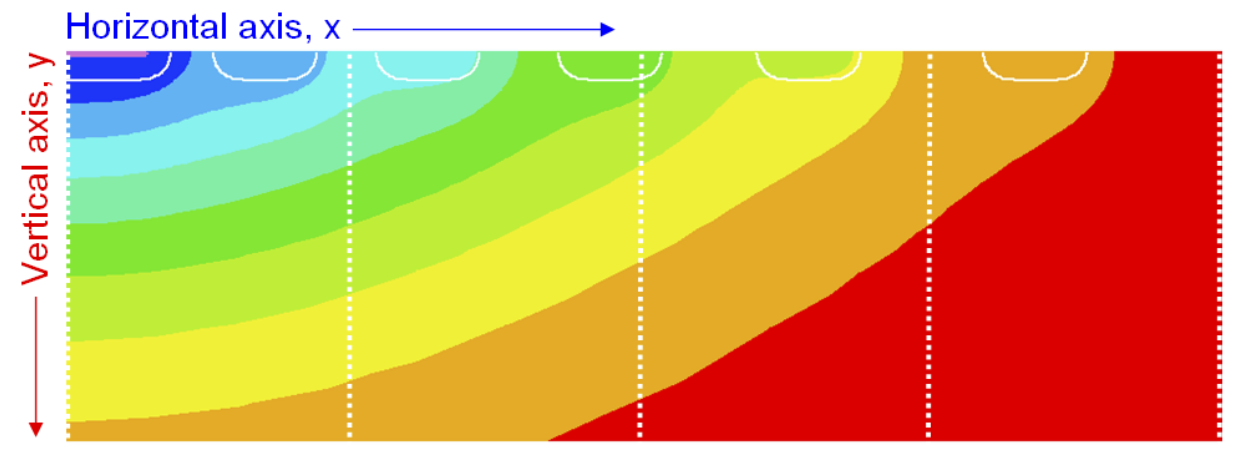

(a)

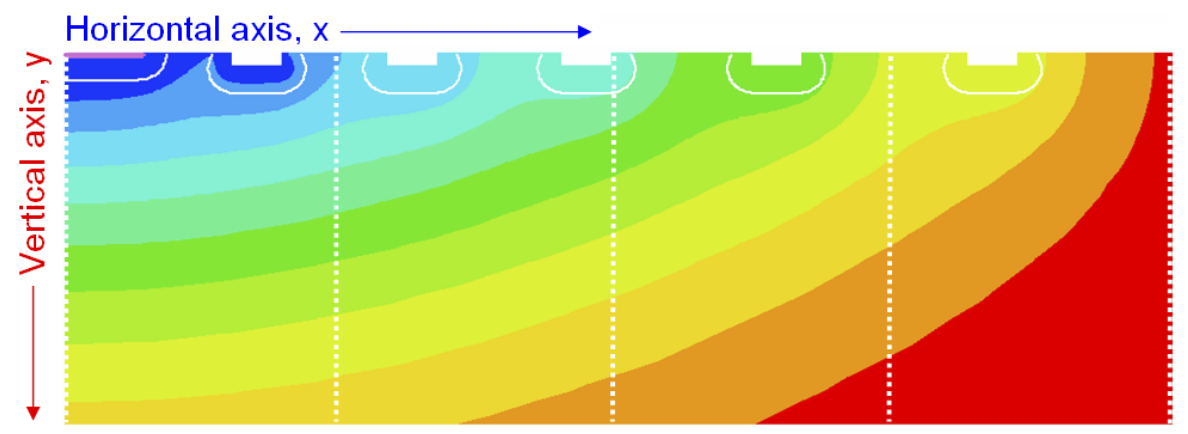

(b)

Fig. 2. (a) Potential distribution for conventional 5-ring termination structure. (BV $=1,725 \mathrm{~V})$ (b) Potential distribution for trenched 5-ring termination structure. $(\mathrm{BV}=2,244 \mathrm{~V})$

The applied voltage is supported in larger area in case of the trenched field-limiting ring, Fig. 2 (b).

The breakdown voltage of conventional structure is $1,725 \mathrm{~V}$ however the breakdown voltage of the $5 \mu \mathrm{m}$ trench etched structure is $2,244 \mathrm{~V}$. In other words, by etching the conventional field limiting ring, nearly $500 \mathrm{~V}$ increased breakdown voltage can be obtained. Moreover, as the trench depth is deeper, the critical electric field depth is also deeper. If the critical electric field occurs near the surface, silicon dioxide layer can be damaged and the interface charges can be generated. These effects degrade the device reliability. Therefore it is expected that the trenched field limiting ring can improve the breakdown voltage characteristics and reliability problems simultaneously. 
Fig. 3 (a) and (b) show the 3-dimensional surface electric field distribution of the two structures when they are under avalanche breakdown condition. In case of the conventional structure, Fig. 3 (a), only inner junctions are supporting the highest critical electric field $\left(2.50 \times 10^{5} \mathrm{~V} / \mathrm{cm}\right)$ and the other rings are not operated well as the voltage divider. On the other hand, in case of $5 \mu \mathrm{m}$ trenched structure, Fig. 7 (b), all junctions are supporting the

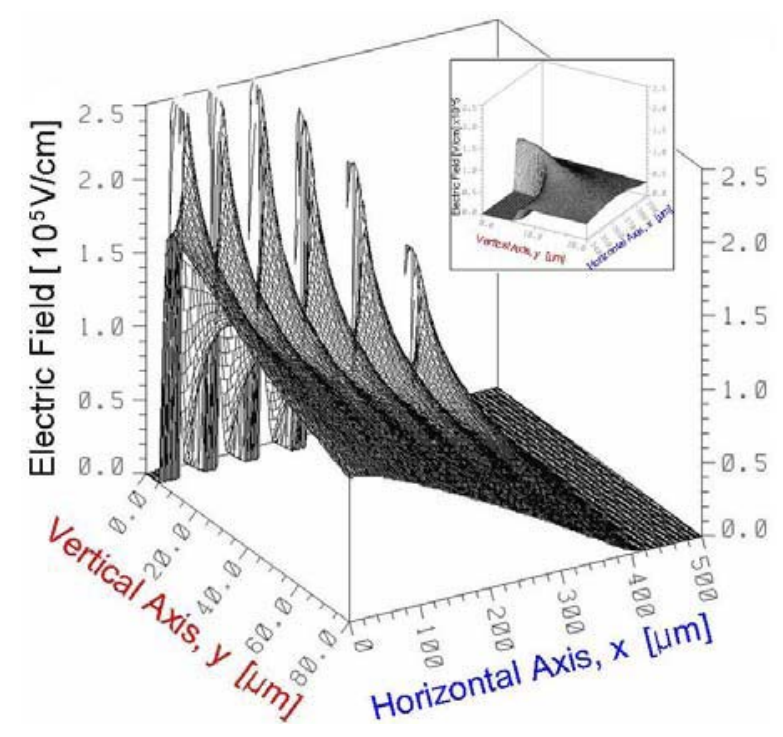

(a)

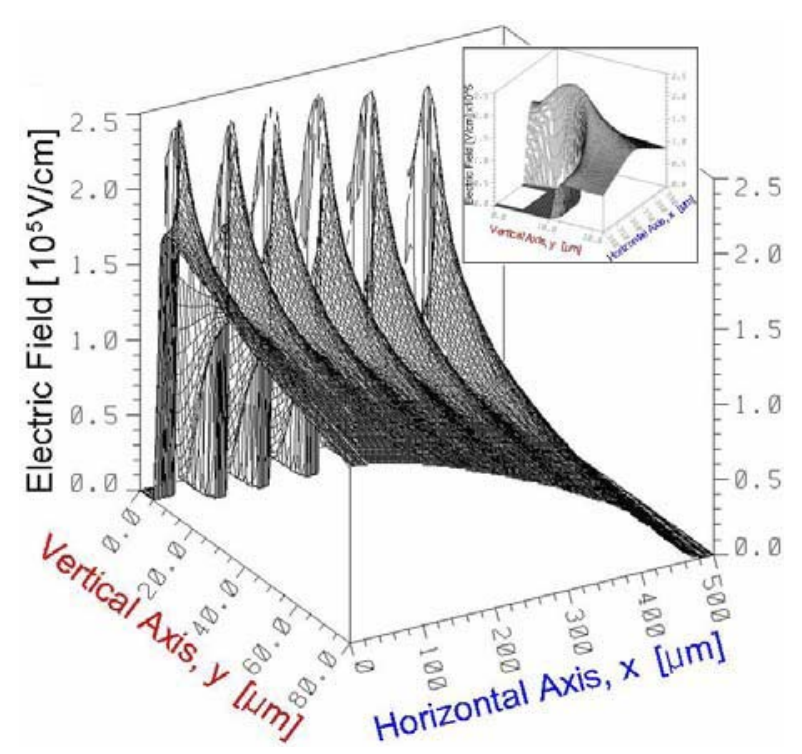

(b)

Fig. 3. (a) Surface electric field distribution of the conventional 5-ring termination structure $\left(\mathrm{E}_{\mathrm{cri}}=\right.$ $2.50 \times 10^{5} \mathrm{~V} / \mathrm{cm}$ ), (b) Surface electric field distribution of the trenched 5-ring termination structure $\left(\mathrm{E}_{\text {cri }}=2.25 \times 10^{5} \mathrm{~V} / \mathrm{cm}\right)$, the insets show the critical electric field point. 
same and lowered critical electric field $\left(2.25 \times 10^{5} \mathrm{~V} / \mathrm{cm}\right)$. This means that the field limiting ring is optimized as a voltage divider and the breakdown voltage can be maximized. The insets in Fig. 3 (a), (b) stand for the critical electric field depth. These insets show the electric field peak are located far from the surface by trench etching.

This effect can lower the charge generation probability and improve the device stability.

\section{Conclusion}

A new edge termination technique to improve breakdown voltage characteristics and reliability problems has been proposed. The newly proposed structure has deep ring junctions and they can be obtained by trenching the field limiting ring site which would be ion-implanted.

The simulation results show that the trenched field limiting ring have smaller critical electric field $\left(2.25 \times 10^{5} \mathrm{~V} / \mathrm{cm}\right)$ in comparison with conventional structure $\left(2.50 \times 10^{5} \mathrm{~V} / \mathrm{cm}\right)$ and this effect leads to near $30 \%$ increase of breakdown voltage. Moreover, the trenched structure has uniform electric field distribution as well as much lower and deeper electric field peaks. In conventional structure, the oxide damages caused by the electric field peak near the surface have been the biggest problem, however the deeper electric field peak of the proposed structure make the field limiting ring technique more stable. These mean that the trenched structure have improved voltage blocking capability and reliability.

By adding one trench etching step, the drawback of the conventional field limiting ring about reliability problems can be compensated and the advantage of voltage blocking capability can be maximized.

\section{Acknowledgments}

This research was supported by a Korea University Grant. 